\title{
Bayesian ensemble approach to error estimation of interatomic potentials
}

Frederiksen, Søren Lund; Jacobsen, Karsten Wedel; Brown, K.S.; Sethna, J.P.

Published in:

Physical Review Letters

Link to article, DOI:

10.1103/PhysRevLett.93.165501

Publication date:

2004

Document Version

Publisher's PDF, also known as Version of record

Link back to DTU Orbit

Citation $(A P A)$ :

Frederiksen, S. L., Jacobsen, K. W., Brown, K. S., \& Sethna, J. P. (2004). Bayesian ensemble approach to error estimation of interatomic potentials. Physical Review Letters, 93(16), 165501.

https://doi.org/10.1103/PhysRevLett.93.165501

\section{General rights}

Copyright and moral rights for the publications made accessible in the public portal are retained by the authors and/or other copyright owners and it is a condition of accessing publications that users recognise and abide by the legal requirements associated with these rights.

- Users may download and print one copy of any publication from the public portal for the purpose of private study or research.

- You may not further distribute the material or use it for any profit-making activity or commercial gain

- You may freely distribute the URL identifying the publication in the public portal 


\title{
Bayesian Ensemble Approach to Error Estimation of Interatomic Potentials
}

\author{
Søren L. Frederiksen and Karsten W. Jacobsen \\ CAMP, Department of Physics, Technical University of Denmark, DK-2800 Kongens Lyngby, Denmark
}

Kevin S. Brown and James P. Sethna

Laboratory of Atomic and Solid State Physics (LASSP), Clark Hall, Cornell University, Ithaca, New York 14853-2501, USA

(Received 18 December 2003; published 15 October 2004)

\begin{abstract}
Using a Bayesian approach a general method is developed to assess error bars on predictions made by models fitted to data. The error bars are estimated from fluctuations in ensembles of models sampling the model-parameter space with a probability density set by the minimum cost. The method is applied to the development of interatomic potentials for molybdenum using various potential forms and databases based on atomic forces. The calculated error bars on elastic constants, gamma-surface energies, structural energies, and dislocation properties are shown to provide realistic estimates of the actual errors for the potentials.
\end{abstract}

DOI: 10.1103/PhysRevLett.93.165501

Interatomic potentials are used extensively to study the structural and dynamical properties of a wide range of materials from biomolecules to polymers and semiconductors to metals and alloys [1]. Typically interatomic potentials are computationally very fast because calculations of atomic energies and forces are carried out by explicit evaluations of pairlike or angular terms which depend exclusively on the coordinates of a few atoms at a time. This is in contrast to the electronic structure or quantum chemistry methods which involve a direct treatment of the electronic degrees of freedom. The interatomic potentials therefore allow for more elaborate simulations: the dynamics of larger systems can be studied for longer times or a more accurate sampling of the configuration space can be obtained in studies of thermal properties.

The speed and simplicity of the interatomic potentials are, however, often obtained at the cost of a lurking uncertainty about their accuracy and predictive power. Most interatomic potentials are constructed in such a way that a number of essential quantities are guaranteed to be reproduced correctly compared to either experimental values or more accurate calculations. For example, interatomic potentials of crystalline metals usually reproduce experimental lattice constants and cohesive energies. However, to what extent a given interatomic potential is transferable (i.e., can be used in more general situations) is usually a matter of experience and not something which is tested systematically. In this Letter we propose a systematic method for estimating transferability errors: through the generation of ensembles of potentials we estimate error bars for the predictions of these atomistic calculations.

In standard potential development a functional form is derived or taken from the literature and its parameters $\theta$ are fitted to a list of data originating either from experiment or from theoretical calculations. By minimizing a cost function $C(\theta)$, which measures the quality of the fit,
PACS numbers: $62.20 .-\mathrm{x}, 02.50 . \mathrm{Ng}, 31.50 . \mathrm{Bc}, 34.20 . \mathrm{Cf}$

the procedure returns a minimum cost $C_{0}$ and the corresponding set of parameters $\theta_{0}$. The best-fit basin can be quite shallow in several parameter directions; many neighboring parameter sets $\theta$ will fit the data almost as well as well as $\theta_{0}$. This leads us to question the result of predictions made using the best-fit parameters alone; it seems dangerous not to consider fluctuations in the predictions generated by neighboring parameter sets.

The approach described in the present Letter is inspired by Bayesian statistics [2] and recent work on the modeling of complex biochemical networks [3]. Whereas the standard method takes a maximum likelihood approach to parameter selection, the method we employ assigns a conditional probability $P(\theta \mid D, M)$ to each set of parameters given a database $D$ and a model $M$. Assuming independent normal errors in the database, this probability may be written as

$$
P(\theta \mid D, M) \propto \exp \left[-\frac{C(\theta)}{T}\right]
$$

with cost function $C(\theta)=\sum_{i}^{N}\left(y_{i}-y_{i}^{\theta}\right)^{2} / 2$. Here $y_{i}$ are quantities from the database, $y_{i}^{\theta}$ are the corresponding quantities calculated using the model, and $T$ is a temperature introduced to formalize the weighting of different parameter sets. Typical data-fitting approaches to potential development can be viewed as a special case of this approach using $T=0$ or equivalently $P(\theta \mid D, M)=$ $\delta\left(\theta-\theta_{0}\right)$. In what follows, we use the minimal value of the $\operatorname{cost} C_{0}$ to set the temperature. Specifically, since each mode contributes an average energy of $T / 2$ in a harmonic model, we define a natural temperature by $T_{0}=2 C_{0} / N_{p}$ where $N_{p}$ is the number of parameters in the potential. At this temperature the distribution given in Eq. (1) samples over parameter sets whose additional errors are within the residual error of the best fit.

Based on the probability in Eq. (1) an ensemble of potential parameters can be generated given a temperature 
$T$, a database $D$, and a model $M$. For any observable $O$ the ensemble mean $\left\langle\left. O\right|_{T, D, M}\right.$ and standard deviation $\left.\sigma_{O}^{2}\right|_{T, D, M}$ can then be calculated.

By generating an entire ensemble of potentials it is possible to explore how uncertainty in the parameters influences predictions of the potential. Usually accuracy and transferability are tested against an external database while with an ensemble it is possible to test these properties internally by making use of fluctuations in the ensemble.

Interatomic potentials for molybdenum.-While the previous discussion has been quite general and applies to any multiparameter model matched to data, in this section we specialize to the development and evaluation of an interatomic potential for molybdenum.

The data used to fit the molybdenum potential will be given in the form of atomic forces. This idea, known as the force matching method, was introduced by Ercolessi and Adams [4] and offers a simple way of parsing data from density functional theory (DFT) calculations into an interatomic potential. A force field database covering bulk molybdenum systems is constructed. Four different periodic systems with 64 atoms in the unit cells are used: a bcc crystal with random atomic displacements picked from a Gaussian distribution of width $0.047 \AA$ (corresponding to roughly a temperature of $300 \mathrm{~K}$ ), a bcc crystal with random displacements of $0.135 \AA$ $(2500 \mathrm{~K})$, a system with two easy-core dislocations, and a system with two hard-core dislocations. In the latter two systems atomic displacements corresponding to $300 \mathrm{~K}$ are also applied. The atomic forces are calculated using the DFT pseudopotential code DACAPO [5].

In the following we test three different potential forms: an effective medium theory potential [10] with angular dependent terms [which will be referred to as the modified effective medium theory (MEMT) potential], a Finnis-Sinclair (FS) potential [11], and a modified embedded atom method (MEAM) [12] potential. The MEMT potential has the usual effective medium form [10] with two modifications. First, the pair potential is not given by a single exponentially decaying function of interatomic distance but is expanded in cosines in a range of interatomic distances, and second angular dependence has been added. The angular dependent contribution to the total energy is in the form of sums over pairs of atoms using projections onto $p$ and $d$ spherical harmonics. Because of the cosine expansions of the pair potential and because of two functions appearing in the angular terms, the MEMT potential has a variable number of parameters. The values for the lattice constant $(a=$ $3.187 \AA)$, the bulk modulus $(B=266 \mathrm{GPa})$ and the cohesive energy $\left(E_{\mathrm{coh}}=-6.029 \mathrm{eV}\right)$ are by construction constrained to the DFT values. The details of the potential will be presented elsewhere [13].

The FS potential has a general form somewhat similar to the MEMT potential without the angular terms, but its detailed functional form is different and has only six free parameters. The MEAM potential differs from the MEMT and FS potentials both in the choice for the angular dependence and in the utilization of a special "screening" method which modifies the interactions between two atoms depending on their environment. Constraining the lattice constant, the bulk modulus, and the cohesive energy gives the MEAM potential only five parameters.

The optimal number of parameters for the MEMT potential is determined using the training-test method [14]. The potential parameters are fitted to a training set while the performance of the potential is evaluated using a test set. Force fields from bcc systems at $2500 \mathrm{~K}$ are used as training and test sets. The training error is a monotonically decreasing function of the number of parameters while the test error has a global minimum as shown in Fig. 1. The optimal number of parameters is set by the minimum test error. The minimum arises as a compromise between an insufficient model (too few parameters) and overfitting of data (too many parameters). A similar approach was reported by Mishin et al. [15].

Potential Ensembles.-The Monte Carlo algorithm offers a simple way of generating an ensemble according to Eq. (1). However, special care has to be taken to obtain an efficient sampling of the parameter space because of the character of the cost function. In the region around the optimal parameter set, $\theta_{0}$, the curvatures of the cost function in different directions vary enormously [3]. In the case of the 21-parameter MEMT potential the eigenvalues of the Hessian span 7 orders of magnitude. The Monte Carlo trial moves are therefore rescaled using the

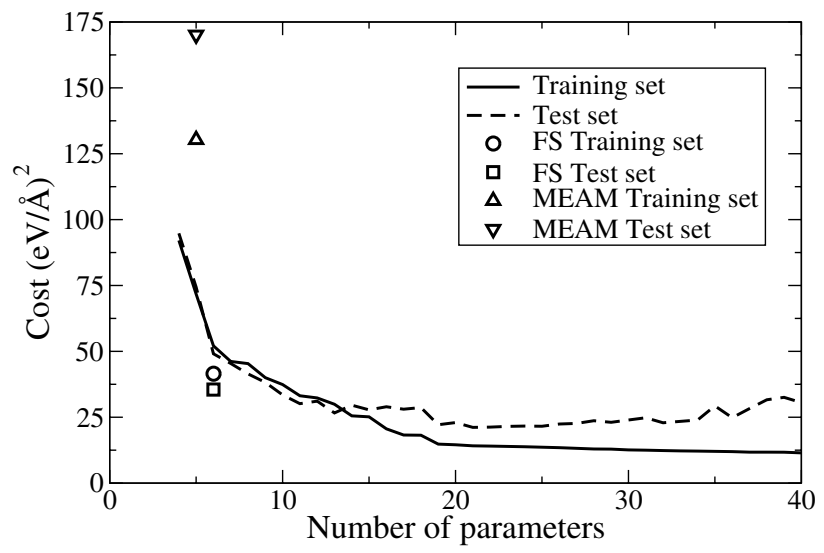

FIG. 1. Model selection using test and training sets. The cost is plotted as function of the total number of potential parameters. MEMT potentials with up to 40 parameter are fitted while the FS and the MEAM have a fixed number of parameters. The minimum test error is obtained with 21 parameters with one parameter in the density function, 9 parameters in the pair potential, 6 parameters in the angular $p$ term, and 5 parameters in the angular $d$ term. The relatively poor performance of the MEAM potential is also seen in tantalum [19]; its screening method is not useful on the bulk systems we have used. 
calculated Hessian as in Ref. [3]. In Fig. 2 the distributions of the structural energy difference between fcc and bcc are plotted for ensembles based on the MEMT potential for different temperatures. The ensembles are formed from 1000 sets of parameters taken from Monte Carlo simulations involving $10^{6}$ trial moves [16]. When the temperature is reduced the distributions contract around the best-fit value. The structural energy difference is one of the quantities which is not particularly well reproduced by the MEMT best-fit potential (compared to, for example, the elastic constants), however, at the temperature $T_{0}$ the width of the ensemble distribution is seen to become comparable to the difference between the best-fit value and the DFT value.

In the following we study to what extent the distribution of an observable can be taken as an estimate of the accuracy of the prediction. For a given set of observables $\mathcal{O}$ we define the cumulative error distribution $D$ as

$$
D(r)=\frac{1}{N} \sum_{O \in \mathcal{O}} \Theta\left(r \sigma_{O}-\left|O_{0}-O^{\mathrm{DFT}}\right|\right),
$$

where $\Theta$ is the Heaviside step function and the summation is running over all $N$ observables in the set $\mathcal{O}$. The observable calculated with DFT (i.e., the "correct" result) is denoted by $O^{\mathrm{DFT}}$ while the best-fit value is denoted by $O_{0}$. The cumulative error distribution at $r$ thus returns the fraction of observables having an error of less than $r \sigma_{O}$. For a Gaussian distribution of errors and standard deviations one therefore obtains $D(r)=\operatorname{erf}(r / \sqrt{2})$.

We first study the distribution for the force components of all atoms in a database similar to the one used for generating the potential ensembles. The new database differs from the one used to generate the ensembles only in the displacements of the $4 \times 64$ atoms. Since each atom has three force components the total number of observables is 768. In Fig. 3 the cumulative distributions are

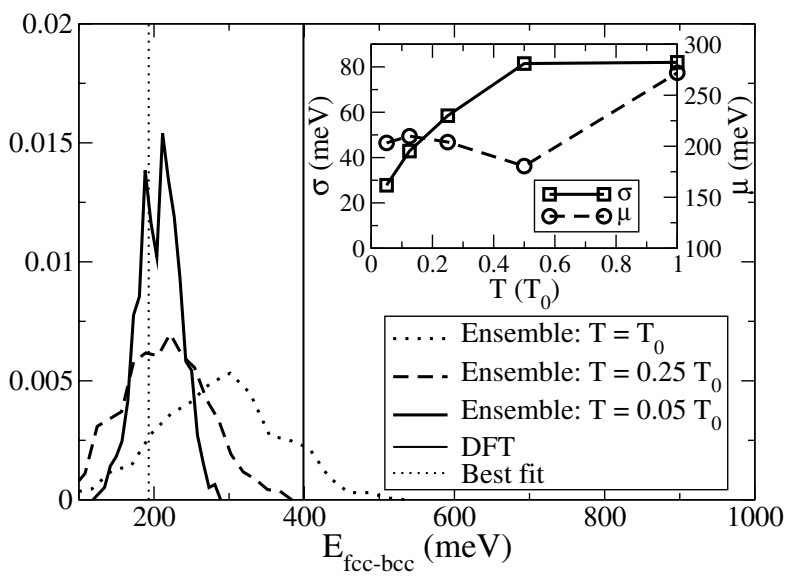

FIG. 2. Ensemble distributions of the structural energy difference between fcc and bcc. Distributions are calculated using the MEMT potential for different ensemble temperatures. In the inset means $(\mu)$ and standard deviations $(\sigma)$ are plotted as function of the temperature. plotted for the three potentials using ensembles generated at a temperature of $T_{0}$. The similarity with the Gaussian error estimate is good for the Finnis-Sinclair and the MEMT potentials. For the MEAM potential which is the most restricted model, we are underestimating the errors but by less than a factor of 2 .

Encouraged by the good agreement for the error estimate on the force components we proceed by studying other observables which are less directly involved when the potentials are generated. In Table I different bulk properties are calculated based on the potential ensembles. We also vary the character of the fitting database used to generate the potentials. The jagged curve in Fig. 3 shows the cumulative distribution using all the means and standard deviations listed in Table I. The properties in Table I were selected and tabulated before Fig. 3 was envisioned. Considering that the error estimates range from $7 \%$ to above $200 \%$, the fit of the ratios with the true errors to a Gaussian is remarkable. We note that the two largest ratios are both again for the MEAM potential, where our error estimates for the forces were also slightly too small.

It can be noted that the anharmonic effects in the ensembles at $T_{0}$ can be quite strong, showing up, for example, as considerable differences between best-fit values and ensemble averages. In some case potentials in the $T_{0}$ ensembles also appear "unphysical" in the sense that atomic relaxations may lead to completely different structures. We therefore also tried an alternative procedure for estimating the error bars by performing simulations at a lower temperature of $T_{0} / 4$. In this regime the anharmonic effects turn out to be small and by appropriate harmonic rescaling of the obtained standard deviations to $T_{0}$ an

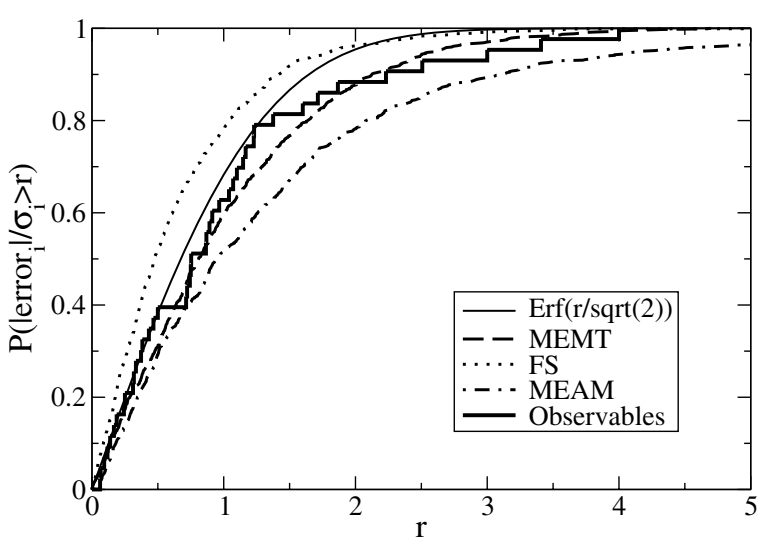

FIG. 3. Cumulative error distributions of force components and the observables in Table I. The cumulative distribution defined in Eq. (2) is calculated for each of the three potentials using the force components as the set of observables. The ensembles are generated at a temperature of $T_{0}$. Furthermore, the distribution obtained with all the observables in Table I is also shown. The good agreement with the Gaussian error function is an indication that the calculated standard deviations provide a good estimate of the actual errors. 
TABLE I. Best-fit values and standard deviations of potential quantities. The standard deviations are calculated from ensembles at the temperature $T_{0}$. The quantities cover the three elastic constants $c_{11}, c_{12}$, and $c_{44}$, the interface energies for the gamma surfaces with (110) and (211) orientations and a displacement of half a Burgers vector in the (1 $\overline{1} \overline{1})$ direction, the energy differences between the bcc structure and the a15 and fcc structures, the energy difference between a screw dislocation in the hard core and easy core structures, and finally the energy required to create a double kink on a screw dislocation. For the first three columns the database includes all the four 64 atom systems described in the text while the "BCC" database includes only the bcc system at $2500 \mathrm{~K}$. The database "Con." denotes the full database with the additional constraints $\Delta E_{\text {Hard-Easy }}=150 \pm 50 \mathrm{meV} / b$ and $c_{44}=90 \pm 10 \mathrm{GPa}$.

\begin{tabular}{|c|c|c|c|c|c|c|c|c|c|c|c|c|}
\hline \multirow{2}{*}{$\begin{array}{c}\text { Pot. } M(\text { Database } D) \\
\text { Observable } O \\
\end{array}$} & \multicolumn{2}{|c|}{ MEMT } & \multicolumn{2}{|c|}{ FS } & \multicolumn{2}{|c|}{ MEAM } & \multicolumn{2}{|c|}{ MEMT (bcc) } & \multicolumn{2}{|c|}{ MEMT (Con.) } & \multirow[t]{2}{*}{ DFT } & \multirow[t]{2}{*}{ Expt. } \\
\hline & $O_{0}$ & $\sigma_{O}$ & $O_{0}$ & $\sigma_{O}$ & $O_{0}$ & $\sigma_{O}$ & $O_{0}$ & $\sigma_{O}$ & $O_{0}$ & $\sigma_{O}$ & & \\
\hline$c_{11}(\mathrm{GPa})$ & 440 & 34 & 456 & 130 & 277 & 49 & 405 & 34 & 440 & 15 & 444 & 450 [17] \\
\hline$c_{12}(\mathrm{GPa})$ & 179 & 18 & 228 & 70 & 272 & 24 & 197 & 18 & 179 & 16 & 176 & $173[17]$ \\
\hline$c_{44}(\mathrm{GPa})$ & 86 & 33 & 89 & 33 & 71 & 25 & 86 & 44 & 86 & 8 & 97 & 125 [17] \\
\hline$\gamma_{(110)}\left(\frac{b}{2}\right)\left(\mathrm{J} / \mathrm{m}^{2}\right)$ & 1.37 & 0.15 & 1.28 & 0.30 & 0.91 & 0.57 & 1.33 & 0.15 & 1.37 & 0.14 & 1.61 & \\
\hline$\gamma_{(211)}\left(\frac{b}{2}\right)\left(\mathrm{J} / \mathrm{m}^{2}\right)$ & 1.54 & 0.15 & 1.48 & 0.34 & 1.10 & 0.68 & 1.51 & 0.14 & 1.54 & 0.14 & 1.61 & \\
\hline$\Delta E_{a 15 \text {-bcc }}(\mathrm{meV} / \mathrm{at})$. & 80 & 61 & 125 & 393 & 318 & 178 & 38 & 81 & 80 & 51 & 99 & \\
\hline$\Delta E_{\mathrm{fcc}-\mathrm{bcc}}(\mathrm{meV} / \mathrm{at})$. & 216 & 82 & 427 & 456 & 207 & 222 & 173 & 313 & 216 & 73 & 399 & \\
\hline$\Delta E_{\text {Hard-Easy }}(\mathrm{eV} / \mathrm{b})$ & 0.27 & 0.14 & 0.40 & 0.29 & -0.23 & 1.10 & 0.33 & 0.23 & 0.27 & 0.05 & 0.12 & \\
\hline$\Delta E_{D \text {-Kink }}(\mathrm{eV})$ & 0.94 & 0.37 & & & & & 1.46 & 0.43 & 0.94 & 0.38 & - & $1.27[18]$ \\
\hline
\end{tabular}

even better agreement with the Gaussian distribution in Fig. 3 is obtained.

In conclusion, we have proposed a general method to evaluate interatomic potentials. Without comparison to measurements or more accurate calculations for a given observable the method is able to assign an error bar to the calculated value. Of course, in order to validate the approach, we have focused on quantities whose results are already known either from DFT or experiments, thus allowing us to compare potentials in a systematic way. The method can be used to evaluate different types of interatomic potentials against each other and may also be useful in guiding the choice of functional forms when constructing new potentials. It may also be possible to use the approach to optimize the fitting database when generating potentials with specific applications in mind.

We would like to thank Nick Bailey and Valerie Coffman for implementing the MEAM potential for us. We furthermore acknowledge support from NSF Grant No. DMR-0218475, from the Materials Research Program of the Danish Research Agency Grant No. 5020-00-0012, and from the Danish Center for Scientific Computing.

[1] M. Finnis, Interatomic Forces in Condensed Matter (Oxford University Press, Oxford, 2003).

[2] E. T. Jaynes, Probability Theory (Cambridge University Press, Cambridge, United Kingdom, 2003).

[3] K. S. Brown and J. P. Sethna, Phys. Rev. E 68, 021904 (2003).

[4] F. Ercolessi and J. B. Adams, Europhys. Lett. 26, 583 (1994).

[5] Further details about the DFT calculations: DACAPO is freely available at http://www.fysik.dtu.dk/campos/ Ion cores are represented by ultrasoft pseudopotentials [6]. Exchange and correlation are calculated within the generalized gradient approximation (Perdew et al. [7]). All calculations are performed in supercells with periodic boundary conditions. Kohn-Sham wave functions and densities are expanded on plane waves with cutoffs of 340 and $500 \mathrm{eV}$, respectively. $k$ points are sampled using a Monkhorst-Pack grid. For further references, see Refs. [8,9].

[6] D. Vanderbilt, Phys. Rev. B 41, 7892 (1990).

[7] J. P. Perdew, J. A. Chevary, S. H. Vosko, K. A. Jackson, M. R. Pederson, D. J. Singh, and C. Fiolhais, Phys. Rev. B 46, 6671 (1992).

[8] S. R. Bahn and K.W. Jacobsen, Comput. Sci. Eng. 4, 56 (2002).

[9] B. Hammer, L. B. Hansen, and J. K. Nørskov, Phys. Rev. B 59, 7413 (1999).

[10] K.W. Jacobsen, P. Stoltze, and J. K. Nørskov, Surf. Sci. 366, 394 (1996).

[11] M.W. Finnis and J. E. Sinclair, Philos. Mag. A 50, 45 (1984).

[12] M. I. Baskes, Phys. Rev. B 46, 2727 (1992).

[13] S. L. Frederiksen, K.W. Jacobsen, K. S. Brown, and J. P. Sethna (to be published).

[14] T. Hastie, R. Tibshirani, and J. Friedman, The Elements of Statistical Learning (Springer, New York, 2001).

[15] Y. Mishin, D. Farkas, M. J. Mehl, and D. A. Papaconstantopoulos, Phys. Rev. B 59, 3393 (1999).

[16] In the case of the FS potentials two potentials had to be excluded due to nonsensical results related to the neighbor-list treatment in our MD program.

[17] G. Simmons and H. Wang, Single Crystal Elastic Constants and Calculated Aggregate Proporties (The M.I.T. Press, Cambridge, Massachusetts, 1971).

[18] L. Hollang, M. Hommel, and A. Seeger, Phys. Status Solidi A 160, 329 (1997).

[19] Y. Li, D. J. Siegel, J. B. Adams, and X.-Y. Liu, Phys. Rev. B 67, 125101 (2003). 